\title{
Analysis of clinical characteristics, laboratory findings and therapy for 134 cases of COVID-19: a retrospective analysis
}

Rui Zhang

Wuhan Xinzhou District People's Hospital

Jie Zhang ( $\triangle 945128911 @ q q . c o m$ )

Xinzhou District People's Hospital『Wuhan, CHina

Weining Chen

Wuhan Xinzhou District People's Hospital

Xiaolin Li

Wuhan Xinzhou District People's Hospital

Jiebing Chen ( $3510352814 @ q q . c o m$ )

Wuhan Xinzhou Disrtict People's Hospital

\section{Research Article}

Keywords: SARS-CoV-2, COVID-19, clinical characteristics, laboratory findings, therapy, gamma globulin

Posted Date: November 6th, 2020

DOI: https://doi.org/10.21203/rs.3.rs-83029/v2

License: (c) (i) This work is licensed under a Creative Commons Attribution 4.0 International License. Read Full License 


\section{Abstract}

Background: The coronavirus disease 2019 (COVID-19) pandemic has spread worldwide. As of October $27^{\text {th }}, 2020$, the number of worldwide laboratory-confirmed cases had reached $43,340,710$, with $1,157,496$ deaths. We sought to analyze the clinical characteristics, laboratory findings and therapy for a set of COVID-19 cases.

Methods: For this retrospective study, we extracted data for 134 patients with laboratory-confirmed COVID-19 at our hospital from January $16^{\text {th }}$ to April $24^{\text {th }}, 2020$. Cases were confirmed by real-time RT-PCR and abnormal radiologic findings. Outcomes were followed up until May $1^{\text {st }}, 2020$.

Results: An outcome of death or severe COVID-19 was more likely to occur with coinfection and severe underlying diseases. Age above 60 years old, male sex and symptoms such as fever, cough, chest tightness, headaches and fatigue were also related to severe COVID-19 and an outcome of death. In addition, high temperature, high blood leukocyte and neutrophil counts, C-reactive protein and D-dimer levels, and alanine aminotransferase, aspartate aminotransferase, a-hydroxybutyrate dehydrogenase, lactate dehydrogenase and creatine kinase activities were related to severe COVID-19 and an outcome of death, as was a low lymphocyte count. The administration of gamma globulin appeared to be helpful for reducing mortality in patients with severe COVID-19; however, as the $\mathrm{P}$ value was greater than 0.05 $(P=0.180)$, studies of the same conditions with larger samples are needed.

Conclusion: Multiple factors are related to severe COVID-19 and an outcome of death. The administration of gamma globulin seemed to be helpful for reducing mortality in severe cases. More related studies are needed in the future.

\section{Clinical trial registration}

Not applicable

\section{Introduction}

The COVID-19 (coronavirus disease 2019) pandemic, which is caused by SARS-CoV-2 (severe acute respiratory syndrome coronavirus 2), has spread worldwide. According to information from the official website of the World Health Organization, the number of laboratory-confirmed cases reached 43,340,710 as of October $27^{\text {th }}, 2020$, with $1,157,496$ deaths, worldwide.

In the past several months, many studies have described the clinical characteristics of and laboratory findings for patients with COVID-19. ${ }^{1-3}$ When assessing the relationship between potential risk factors and COVID-19 severity, some studies have utilized an indicator of the highest or lowest level during hospitalization, whereas other studies have mainly used an indicator with a fixed value on admission. ${ }^{1-3}$ This raises the following questions: what is the sphere of application and clinical significance of such indicators, and what is the relationship between them? To address these questions, we used both the 
fixed value on admission and the highest or lowest level of candidate indicators during hospitalization to assess the relationship between potential risk factors and COVID-19 severity. Herein, we highlight risk factors related to severe COVID-19 and an outcome of death and explore the clinical significance of these risk factors and the role of the two types of indicators.

\section{Methods}

\subsection{Data sources}

We performed a retrospective study on the clinical characteristics, laboratory findings and therapy for laboratory-confirmed cases of COVID-19.

Inclusion criteria:

1. All patients were diagnosed with pneumonia based on clinical manifestations and abnormal findings of chest X-ray or computed tomography.

2. A confirmed case of COVID-19 was defined as a positive result by high-throughput sequencing or real-time reverse-transcriptase polymerase-chain-reaction assay using nasal and pharyngeal swab specimens.

Exclusion criteria:

1. Patients with infected with common bacteria or viruses associated with community-acquired pneumonia.

2. Patients with severe underlying disease.

3. Procalcitonin level $>0.5 \mathrm{ng} / \mathrm{ml}$.

A flow chart, from the total number of patients to the 134 patients included in the study, is shown in Figure 1. Fourteen patients with severe underlying disease (i.e., chronic lung disease, chronic heart disease, chronic liver disease, chronic kidney disease) were excluded. Twenty-two patients coinfected with COVID-19 and other respiratory pathogens (i.e., bacteria, Chlamydia pneumoniae, Mycoplasma pneumoniae, adenovirus, or respiratory syncytial virus) associated with community-acquired pneumonia were also excluded, as were 16 patients with both severe underlying diseases and other respiratory pathogen infections. Ultimately, 134 confirmed cases of COVID-19 were included in our study.

All data, including age, sex, temperature and laboratory findings, were extracted from electronic medical records. Laboratory assessments consisted of complete blood count, blood chemistry, coagulation test, liver and renal function, C-reactive protein, a-hydroxybutyrate dehydrogenase activity, lactate dehydrogenase activity and creatine kinase activity. The severity of COVID-19 was defined according to 
the sixth edition of the diagnosis and treatment program of COVID-19 issued by the National Health Commission of the People's Republic of China. This study evaluated 100 nonsevere and 34 severe cases.

\subsection{Definition of severe COVID-19}

The definition of COVID-19 included the following: respiration rate $\geq 30$ times/min; at rest, oxygen saturation $\leq 93 \%$; arterial partial pressure of oxygen $(\mathrm{PaO} 2) /$ fraction of inspired oxygen $(\mathrm{FiO} 2) \leq 300$ $\mathrm{mmHg}$.

\subsection{Laboratory tests}

Laboratory tests were performed every two days or when the health condition of the patient changed.

\subsection{Therapy with gamma globulin}

Administration of gamma globulin was not used as a conventional therapy. Administration depended on the wish of the patients and their relatives.

\subsection{Statistical analysis}

Continuous variables are expressed as medians and interquartile ranges. Categorical variables are summarized as counts and percentages. We grouped patients into severe and nonsevere groups according to the sixth edition of the diagnosis and treatment program of COVID-19 issued by the National Health Commission of the People's Republic of China. Wilcoxon rank-sum tests were applied for continuous variables, and chi-square tests and Fisher's exact tests were used for categorical variables, as appropriate. All analyses were conducted with SPSS software version 23.0 (Statistical Product and Service Solutions). Differences with $\mathrm{P}$ values $<0.05$ were considered significant.

\subsection{Statement of Ethics}

The Ethics Committee of Xinzhou District People's Hospital approved this study.

\subsection{Informed consent}

Not applicable

\section{Results}

\subsection{Coinfection and severe underlying disease}

All 186 patients were divided into two groups, namely, the COVID-19 only group and COVID-19 + severe underlying disease or other respiratory pathogen infections group, as shown in Table 1. 
Table 1. Comparison between COVID-19 only group and COVID-19 + severe underlying disease or other respiratory pathogen infections group

\begin{tabular}{|c|c|c|c|c|c|c|c|}
\hline \multirow[b]{2}{*}{ Groups-No., \% } & \multirow{2}{*}{$\begin{array}{l}\text { All } \\
\text { patients } \\
\quad(n=186)\end{array}$} & \multicolumn{2}{|c|}{ Disease severity } & \multirow[b]{2}{*}{ P1 } & \multicolumn{3}{|c|}{ Outcome } \\
\hline & & $\begin{array}{r}\text { No-severe } \\
(n=123)\end{array}$ & $\begin{array}{l}\text { Severe } \\
(n=63)\end{array}$ & & $\begin{array}{l}\text { Survival } \\
(n=166)\end{array}$ & $\begin{array}{l}\text { Death } \\
(n=20)\end{array}$ & P2 \\
\hline \multirow[t]{2}{*}{ COVID-19 only $(n=134)$} & $\begin{array}{l}134 / 186 \\
(66.1)\end{array}$ & $\begin{array}{c}100 / 123 \\
(81.3)\end{array}$ & $\begin{array}{l}34 / 63 \\
(54.0)\end{array}$ & {$[0.001$} & $\begin{array}{c}128 / 166 \\
(77.1)\end{array}$ & $\begin{array}{c}6 / 20 \\
(30.0)\end{array}$ & $\square 0.001$ \\
\hline & & $\begin{array}{l}100 / 134 \\
(74.6)\end{array}$ & $\begin{array}{l}34 / 134 \\
(25.4)\end{array}$ & & $\begin{array}{c}128 / 134 \\
(95.5)\end{array}$ & $\begin{array}{c}6 / 134 \\
(4.5)\end{array}$ & \\
\hline \multirow[t]{2}{*}{$\begin{array}{l}\text { COVID-19 + severe underlying disease or } \\
\text { other respiratory pathogen infections }(n=52)\end{array}$} & $\begin{array}{c}52 / 186 \\
(33.9)\end{array}$ & $\begin{array}{l}23 / 123 \\
(18.7)\end{array}$ & $\begin{array}{l}29 / 63 \\
(46.0)\end{array}$ & & $\begin{array}{l}38 / 166 \\
(22.9)\end{array}$ & $\begin{array}{l}14 / 20 \\
(70.0)\end{array}$ & \\
\hline & & $\begin{array}{l}23 / 52 \\
(44.2)\end{array}$ & $\begin{array}{l}29 / 52 \\
(55.8)\end{array}$ & & $\begin{array}{l}38 / 52 \\
(73.1)\end{array}$ & $\begin{array}{l}14 / 52 \\
(26.9)\end{array}$ & \\
\hline
\end{tabular}

P values denote comparison between included cases and excluded cases.

In the COVID-19 only group, $25.4 \%$ of the patients had severe cases, and $4.5 \%$ of the patients died. In the COVID-19 + severe underlying disease or other respiratory pathogen infections group, $55.8 \%$ of the patients had severe cases, and $26.9 \%$ of the patients died. (P1ه0.001, P2ख0.001)

Obviously, coinfection and severe underlying disease were related to severe COVID-19 and an outcome of death.

\subsection{Demographic and clinical characteristics}

The demographic and clinical characteristics are presented in Table 2, summarizing 134 cases.

Table 2. Clinical characteristics of 134 patients with COVID-19 


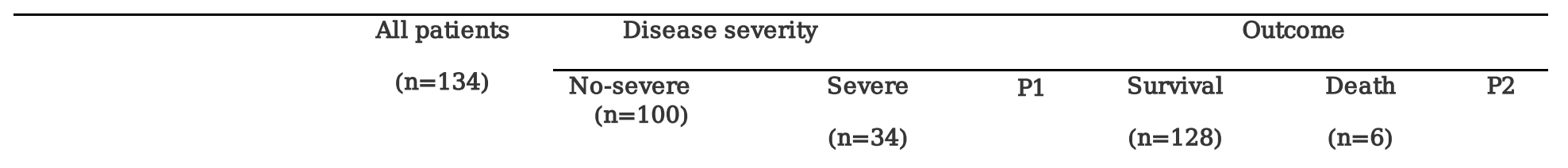

\begin{tabular}{|c|c|c|c|c|c|c|c|}
\hline $\begin{array}{l}\text { Age, Median (range)- } \\
\text { years }\end{array}$ & $\begin{array}{l}49.0(38.7- \\
57.2)\end{array}$ & $48.5(38.0-57.0)$ & $\begin{array}{l}51.0(39.0- \\
59.7)\end{array}$ & 0.292 & $\begin{array}{l}49.0(38.2- \\
57.0)\end{array}$ & $\begin{array}{c}66.0(38.2- \\
76.7)\end{array}$ & 0.078 \\
\hline Age groups-No., \% & & & & 0.332 & & & 0.006 \\
\hline \multirow[t]{2}{*}{$\square 30$ years $(n=16)$} & $16 / 134(11.9)$ & $14 / 100(14.0)$ & $2 / 34(5.9)$ & & $\begin{array}{c}16 / 128 \\
(12.5)\end{array}$ & $0 / 6(0.0)$ & \\
\hline & & $14 / 16(87.5)$ & 2/16 (12.5) & & $\begin{array}{c}16 / 16 \\
(100.0)\end{array}$ & $0 / 16(0.0)$ & \\
\hline \multirow[t]{2}{*}{$30-60$ years $(n=94)$} & $94 / 134(70.1)$ & $70 / 100(70.0)$ & $24 / 34(70.6)$ & & $\begin{array}{c}92 / 128 \\
(71.9)\end{array}$ & $2 / 6(33.3)$ & \\
\hline & & $70 / 94(74.5)$ & $24 / 94(25.5)$ & & $92 / 94(97.9)$ & $2 / 94(2.1)$ & \\
\hline \multirow[t]{2}{*}{$\square 60$ years $(n=24)$} & $24 / 134(17.9)$ & $16 / 100(16.0)$ & $8 / 34(23.5)$ & & $\begin{array}{c}20 / 128 \\
(15.6)\end{array}$ & $4 / 6(66.7)$ & \\
\hline & & $16 / 24(66.7)$ & 8/24 (33.3) & & $20 / 24(83.3)$ & $4 / 24(16.7)$ & \\
\hline Sex -No., \% & & & & 0.549 & & & 0.034 \\
\hline \multirow[t]{2}{*}{ Female $(n=59)$} & $59 / 134(44.0)$ & $46 / 100(46.0)$ & $13 / 34(38.2)$ & & $\begin{array}{c}59 / 128 \\
(46.1)\end{array}$ & $0 / 6(0.0)$ & \\
\hline & & $46 / 59(78.0)$ & $13 / 59(22.0)$ & & $\begin{array}{l}59 / 59 \\
(100.0)\end{array}$ & $0 / 59(0.0)$ & \\
\hline \multirow[t]{2}{*}{ Male $(n=75)$} & $75 / 134(56.0)$ & $54 / 100(54.0)$ & $21 / 34$ (61.8) & & $\begin{array}{c}69 / 128 \\
(53.9)\end{array}$ & $6 / 6(100.0)$ & \\
\hline & & $54 / 75(72.0)$ & $21 / 75(28.0)$ & & $69 / 75(92.0)$ & $6 / 75(8.0)$ & \\
\hline \multicolumn{8}{|l|}{ Symptoms } \\
\hline Fever -No., \% & & & & 0.014 & & & 0.343 \\
\hline \multirow[t]{2}{*}{ With $(n=106)$} & 106/134 (79.1) & $74 / 100(74.0)$ & $32 / 34(94.1)$ & & $\begin{array}{l}100 / 128 \\
(78.1)\end{array}$ & $6 / 6(100.0)$ & \\
\hline & & $74 / 106(69.8)$ & $\begin{array}{l}32 / 106 \\
(30.2)\end{array}$ & & $\begin{array}{c}100 / 106 \\
(94.3)\end{array}$ & $6 / 106(5.7)$ & \\
\hline \multirow[t]{2}{*}{ Without $(\mathrm{n}=28)$} & 28/134 (20.9) & $26 / 100(0.26)$ & $2 / 34(5.9)$ & & $\begin{array}{c}28 / 128 \\
(21.9)\end{array}$ & $0 / 6(0.0)$ & \\
\hline & & $26 / 28(92.9)$ & $2 / 28(7.1)$ & & $\begin{array}{l}28 / 28 \\
(100.0)\end{array}$ & $0 / 28(0.0)$ & \\
\hline \multicolumn{2}{|c|}{ Highest temperature, median (range) - ${ }^{\circ} \mathrm{C}$} & 37.9 (37.1-38.8) & $\begin{array}{l}39.0(38.1- \\
39.5)\end{array}$ & $\square 0.001$ & $\begin{array}{l}38.0(37.2- \\
39.0)\end{array}$ & $\begin{array}{l}39.0(38.9- \\
39.1)\end{array}$ & 0.036 \\
\hline $\begin{array}{l}\text { Highest temperatur } \\
\text { hospitalization-No., \% }\end{array}$ & during & & & 0.001 & & & 0.023 \\
\hline \multirow[t]{2}{*}{$\leq 37^{\circ} \mathrm{C}(\mathrm{n}=26)$} & $26 / 134(19.4)$ & $24 / 100(24.0)$ & $2 / 34(5.9)$ & & $\begin{array}{c}26 / 128 \\
(20.3)\end{array}$ & $0 / 6(0.0)$ & \\
\hline & & $24 / 26(92.3)$ & $2 / 26(7.7)$ & & $\begin{array}{l}26 / 26 \\
(100.0)\end{array}$ & $0 / 26(0.0)$ & \\
\hline \multirow[t]{2}{*}{$37-38{ }^{\circ} \mathrm{C}(\mathrm{n}=39)$} & $39 / 134(29.1)$ & $33 / 100(33.0)$ & 6/34 (17.6) & & $\begin{array}{c}39 / 128 \\
(30.5)\end{array}$ & $0 / 6(0.0)$ & \\
\hline & & $33 / 39(84.6)$ & $6 / 39(15.4)$ & & $39 / 39$ & $0 / 39(0.0)$ & \\
\hline
\end{tabular}




\begin{tabular}{|c|c|c|c|c|c|c|c|}
\hline & & & & & $(100.0)$ & & \\
\hline \multirow[t]{2}{*}{$38-39{ }^{\circ} \mathrm{C}(\mathrm{n}=30)$} & $30 / 134(22.4)$ & $23 / 100(23.0)$ & $7 / 34$ (20.6) & & $\begin{array}{c}29 / 128 \\
(22.7)\end{array}$ & 1/6 (16.7) & \\
\hline & & $23 / 30$ (76.7) & $7 / 30(23.3)$ & & $29 / 30(96.7)$ & 1/30 (3.3) & \\
\hline \multirow[t]{2}{*}{$\geq 39^{\circ} \mathrm{C}(\mathrm{n}=39)$} & $39 / 134$ (29.1) & $20 / 100(20.0)$ & $19 / 34(55.9)$ & & $\begin{array}{c}34 / 128 \\
(26.6)\end{array}$ & 5/6 (83.3) & \\
\hline & & 20/39 (51.3) & $19 / 39$ (48.7) & & $34 / 39$ (87.2) & $5 / 39$ (12.8) & \\
\hline Cough-No., \% & & & & 0.032 & & & 0.337 \\
\hline \multirow[t]{2}{*}{ With $(n=104)$} & $104 / 134(77.6)$ & $73 / 100(73.0)$ & $31 / 34(91.2)$ & & $\begin{array}{c}98 / 128 \\
(76.6)\end{array}$ & $6 / 6(100.0)$ & \\
\hline & & 73/104 (70.2) & $\begin{array}{c}31 / 104 \\
(29.8)\end{array}$ & & $\begin{array}{c}98 / 104 \\
(94.2)\end{array}$ & $6 / 104(5.8)$ & \\
\hline \multirow[t]{2}{*}{ Without $(\mathrm{n}=30)$} & $30 / 134(22.4)$ & $27 / 100(27.0)$ & 3/34 (8.8) & & $\begin{array}{c}30 / 128 \\
(23.4)\end{array}$ & $0 / 6(0.0)$ & \\
\hline & & $27 / 30(90.0)$ & $3 / 30(10.0)$ & & $\begin{array}{c}30 / 30 \\
(100.0)\end{array}$ & $0 / 6(0.0)$ & \\
\hline Chest tightness -No., \% & & & & $\square 0.001$ & & & 0.006 \\
\hline \multirow[t]{2}{*}{ With $(n=59)$} & $59 / 134(44.0)$ & $31 / 100(31.0)$ & $28 / 34(82.4)$ & & $\begin{array}{c}53 / 128 \\
(41.4)\end{array}$ & $6 / 6(100.0)$ & \\
\hline & & $31 / 59$ (52.5) & $28 / 59(47.5)$ & & $53 / 59(89.8)$ & $6 / 59(10.2)$ & \\
\hline \multirow[t]{2}{*}{ Without $(n=75)$} & $75 / 134(56.0)$ & $69 / 100(69.0)$ & $6 / 34(17.6)$ & & $\begin{array}{c}75 / 128 \\
(58.6)\end{array}$ & $0 / 6(0.0)$ & \\
\hline & & $69 / 75(92.0)$ & $6 / 75(8.0)$ & & $\begin{array}{c}75 / 75 \\
(100.0)\end{array}$ & $0 / 75(0.0)$ & \\
\hline Headaches-No., \% & & & & {$[0.001$} & & & 0.019 \\
\hline \multirow[t]{2}{*}{ With $(n=15)$} & 15/134 (11.2) & $5 / 100(5.0)$ & $10 / 34(29.4)$ & & $12 / 128(9.4)$ & $3 / 6(50.0)$ & \\
\hline & & $5 / 15(33.3)$ & $10 / 15(66.7)$ & & $12 / 15(80.0)$ & $3 / 15(20.0)$ & \\
\hline \multirow[t]{2}{*}{ Without $(\mathrm{n}=119)$} & $119 / 134(88.8)$ & $95 / 100(95.0)$ & $24 / 34(70.6)$ & & $\begin{array}{c}116 / 128 \\
(90.6)\end{array}$ & $3 / 6(50.0)$ & \\
\hline & & 95/119 (79.8) & $\begin{array}{c}24 / 119 \\
(20.2)\end{array}$ & & $\begin{array}{c}116 / 119 \\
(97.5)\end{array}$ & $3 / 119(2.5)$ & \\
\hline Fatigue -No., \% & & & & $\square 0.001$ & & & 0.081 \\
\hline \multirow[t]{2}{*}{ With $(n=81)$} & $81 / 134(60.4)$ & $49 / 100(49.0)$ & $32 / 34(94.1)$ & & $\begin{array}{c}75 / 128 \\
(58.6)\end{array}$ & $6 / 6(100.0)$ & \\
\hline & & $49 / 81(60.5)$ & $32 / 81(39.5)$ & & $75 / 81(92.6)$ & 6/81 (7.4) & \\
\hline \multirow[t]{2}{*}{ Without $(n=53)$} & $53 / 134(39.6)$ & $51 / 100(51.0)$ & 2/34 (5.9) & & $\begin{array}{c}53 / 128 \\
(41.4)\end{array}$ & $0 / 6(0.0)$ & \\
\hline & & $51 / 53(96.2)$ & $2 / 53(3.8)$ & & $\begin{array}{c}53 / 53 \\
(100.0)\end{array}$ & $0 / 53(0.0)$ & \\
\hline Diarrhea-No., \% & & & & 0.418 & & & 1.000 \\
\hline \multirow[t]{2}{*}{ With $(n=8)$} & 8/134 (6.0) & $5 / 100(5.0)$ & $3 / 34$ (8.8) & & $8 / 128$ (6.3) & $0 / 6(0.0)$ & \\
\hline & & $5 / 8(62.5)$ & $3 / 8(37.5)$ & & 8/8 (100.0) & $0 / 8(0.0)$ & \\
\hline \multirow[t]{2}{*}{ Without $(\mathrm{n}=126)$} & $126 / 134(94.0)$ & $95 / 100(95.0)$ & $31 / 34$ (91.2) & & $\begin{array}{c}120 / 128 \\
(93.7)\end{array}$ & $6 / 6(100.0)$ & \\
\hline & & $95 / 126(75.4)$ & $\begin{array}{c}31 / 126 \\
(24.6)\end{array}$ & & $\begin{array}{c}120 / 126 \\
(95.2)\end{array}$ & $6 / 126(4.8)$ & \\
\hline
\end{tabular}


P1 values denote comparison between nonsevere and severe cases

P2 values denote comparison between survivors and nonsurvivors

\subsubsection{Age}

We grouped the patients into three groups according to age, as indicated in Table 2.

For the $<30$ years group, $12.5 \%$ of the patients had severe disease, and none of the patients died. For the 30-60 years group, $25.5 \%$ had severe disease, and $2.1 \%$ died. In the $>60$ years group, severe disease developed in $33.3 \%$ of the patients, and $16.7 \%$ died. $(P 1=0.332, P 2=0.006)$

Thus, age $>60$ years was related to an outcome of death.

\subsubsection{Sex}

As depicted in Table $2,44.0 \%$ of the patients were female and $56.0 \%$ male.

In the female group, $22.0 \%$ of the patients developed a severe case, though none of the patients died. For the male group, $28.0 \%$ had severe cases, and $8.0 \%$ died. $(P 1=0.549, \mathrm{P} 2=0.034)$

Overall, male sex was related to an outcome of death.

\subsubsection{Fever}

The data in Table 2 show that $79.1 \%$ of the patients had a fever.

In total, $30.2 \%$ of the patients in the fever group had a severe case of COVID-19, and $5.7 \%$ of the patients died. Among those without fever, $7.1 \%$ had severe disease, and none of the patients died. (P1=0.014, $\mathrm{P} 2=0.343)$

Fever group was found to be related to severe COVID-19.

\subsubsection{Temperature}

As shown in Table 2, we grouped patients into four groups according to their highest temperature during hospitalization.

COVID-19 severity and mortality according to temperature was recorded as follows: $7.7 \%$ and none in the $\leq 37^{\circ} \mathrm{C}$ group; $15.4 \%$ and none in the $37-38^{\circ} \mathrm{C}$ group; $23.3 \%$ and $3.3 \%$ in the $38-39^{\circ} \mathrm{C}$ group; and $48.7 \%$ and $12.8 \%$ in the $\geq 39^{\circ} \mathrm{C}$ group, respectively. $(P 1=0.001, P 2=0.023)$

These data show that a higher temperature was related to severe COVID-19 and an outcome of death. 


\subsubsection{Cough}

Table 2 shows that $77.6 \%$ of the patients had a cough.

For those with cough group, $29.8 \%$ of the patients had a severe case, and $5.8 \%$ died. For those without cough, $10.0 \%$ had severe illness, and none died. $(P 1=0.032, P 2=0.337)$

Clearly, cough was related to severe COVID-19.

\subsubsection{Chest tightness}

As shown in Table $2,44.0 \%$ of the patients had chest tightness.

In total, $47.5 \%$ of the patients in the chest tightness group had severe cases, with $10.2 \%$ mortality; $8.0 \%$ of the patients without chest tightness had severe cases, and none of them died. (P1『0.001, P2=0.006)

Chest tightness was related to severe COVID-19 disease and death.

\subsubsection{Headaches}

As shown in Table $2,11.2 \%$ of the patients experienced headaches.

Among those with headaches, $66.7 \%$ were severe cases, and $20.0 \%$ of the patients died. For those without headaches, $20.2 \%$ were severe cases, and $2.5 \%$ died. (P1ه0.001, $P 2=0.019)$

This study found headache to be related to severe COVID-19 and death.

\subsubsection{Fatigue}

In total, $60.4 \%$ of the patients had fatigue (Table 2 ).

Severe cases comprised $39.5 \%$ of the fatigue group, with $7.4 \%$ dying, and $3.8 \%$ of the without fatigue group, with no deaths. $(\mathrm{P} 1 \otimes 0.001, \mathrm{P} 2=0.081)$

Fatigue was related to severe COVID-19.

\subsubsection{Diarrhea}

As described in Table 2, $6.0 \%$ of the patients had diarrhea.

A total of $37.5 \%$ of the patients with the diarrhea had severe COVID- 19 , though none died; $24.6 \%$ of the patients without diarrhea had severe disease, and $4.8 \%$ died.

In this case, the $\mathrm{P}$ values were greater than 0.05 (P1=0.418, P2=1.000); thus, studies with the same conditions and larger samples are needed. 
The laboratory findings are provided in Tables 3 and 4.

Table 3. Laboratory findings for 134 patients with COVID-19 


\begin{tabular}{|c|c|c|c|c|c|c|c|}
\hline \multirow[b]{2}{*}{ Laboratory findings } & \multirow{2}{*}{$\begin{array}{l}\text { All patients } \\
\qquad(\mathrm{n}=134)\end{array}$} & \multicolumn{2}{|c|}{ Disease severity } & \multicolumn{3}{|c|}{ Outcome } & \multirow[b]{2}{*}{ P2 } \\
\hline & & $\begin{array}{l}\text { No-severe } \\
\quad(n=100)\end{array}$ & $\begin{array}{l}\text { Severe } \\
(n=34)\end{array}$ & $\mathrm{P} 1$ & $\begin{array}{l}\text { Survival } \\
(n=128)\end{array}$ & $\begin{array}{l}\text { Death } \\
(n=6)\end{array}$ & \\
\hline \multicolumn{2}{|c|}{$\begin{array}{l}\text { Highest blood leukocyte count during hospitalization- } \\
\text { No., \% }\end{array}$} & & & $\square 0.001$ & & & $\square 0.001$ \\
\hline \multirow[t]{2}{*}{$\square 10 * 10 \wedge 9 / L(n=27)$} & $27 / 134(20.1)$ & $2 / 100(2.0)$ & $\begin{array}{l}25 / 34 \\
(73.5)\end{array}$ & & $\begin{array}{l}21 / 128 \\
(16.4)\end{array}$ & $\begin{array}{c}6 / 6 \\
(100.0)\end{array}$ & \\
\hline & & $2 / 27(7.4)$ & $\begin{array}{l}25 / 27 \\
(92.6)\end{array}$ & & $21 / 27(77.8)$ & $\begin{array}{l}6 / 27 \\
(22.2)\end{array}$ & \\
\hline \multirow[t]{2}{*}{$4-10^{*} 10^{\wedge} 9 / \mathrm{L}(\mathrm{n}=93)$} & $93 / 134(69.4)$ & $\begin{array}{l}86 / 100 \\
(86.0)\end{array}$ & $7 / 34(20.6)$ & & $\begin{array}{l}93 / 128 \\
(72.7)\end{array}$ & $0 / 6(0.0)$ & \\
\hline & & $\begin{array}{l}86 / 93 \\
(92.5)\end{array}$ & 7/93 (7.5) & & $\begin{array}{c}93 / 93 \\
(100.0)\end{array}$ & $\begin{array}{l}0 / 93 \\
(0.0)\end{array}$ & \\
\hline \multirow[t]{2}{*}{$\square 4^{*} 10^{\wedge} 9 / \mathrm{L}(\mathrm{n}=14)$} & $14 / 134(10.4)$ & $\begin{array}{l}12 / 100 \\
(12.0)\end{array}$ & $2 / 34(5.9)$ & & $\begin{array}{l}14 / 128 \\
(10.9)\end{array}$ & $0 / 6(0.0)$ & \\
\hline & & $\begin{array}{l}12 / 14 \\
(85.7)\end{array}$ & $2 / 14(14.3)$ & & $\begin{array}{c}14 / 14 \\
(100.0)\end{array}$ & $\begin{array}{l}0 / 14 \\
(0.0)\end{array}$ & \\
\hline \multicolumn{2}{|c|}{$\begin{array}{l}\text { Highest neutrophil count during hospitalization-No., } \\
\%\end{array}$} & & & $\square 0.001$ & & & $\square 0.001$ \\
\hline \multirow[t]{2}{*}{$\square 7 * 10^{\wedge} 9 / \mathrm{L}(\mathrm{n}=31)$} & $31 / 134(23.1)$ & $5 / 100(5.0)$ & $\begin{array}{l}26 / 34 \\
(76.5)\end{array}$ & & $\begin{array}{l}25 / 128 \\
(19.5)\end{array}$ & $\begin{array}{c}6 / 6 \\
(100.0)\end{array}$ & \\
\hline & & $5 / 31(16.1)$ & $\begin{array}{l}26 / 31 \\
(83.9)\end{array}$ & & $25 / 31(80.6)$ & $\begin{array}{l}6 / 31 \\
(19.4)\end{array}$ & \\
\hline \multirow[t]{2}{*}{$2-7 * 10^{\wedge} 9 / \mathrm{L}(\mathrm{n}=94)$} & $94 / 134(70.1)$ & $\begin{array}{l}86 / 100 \\
(86.0)\end{array}$ & 8/34 (23.5) & & $\begin{array}{l}94 / 128 \\
(73.4)\end{array}$ & $0 / 6(0.0)$ & \\
\hline & & $\begin{array}{l}86 / 94 \\
(91.5)\end{array}$ & $8 / 94(8.5)$ & & $\begin{array}{c}94 / 94 \\
(100.0)\end{array}$ & $\begin{array}{l}0 / 94 \\
(0.0)\end{array}$ & \\
\hline \multirow[t]{2}{*}{ ( $2 * 10^{\wedge} 9 / \mathrm{L}(\mathrm{n}=9)$} & $9 / 134$ (6.7) & 9/100 (9.0) & $0 / 34(0.0)$ & & $9 / 128(7.0)$ & $0 / 6(0.0)$ & \\
\hline & & 9/9 (100.0) & $0 / 9(0.0)$ & & $9 / 9(100.0)$ & $0 / 9(0.0)$ & \\
\hline \multicolumn{2}{|c|}{$\begin{array}{l}\text { Lowest lymphocyte count during hospitalization-No., } \\
\%\end{array}$} & & & $\square 0.001$ & & & 0.006 \\
\hline \multirow[t]{2}{*}{$\square 0.4^{*} 10^{\wedge} 9 / \mathrm{L}(\mathrm{n}=15)$} & $15 / 134(11.2)$ & $3 / 100(3.0)$ & $\begin{array}{l}12 / 34 \\
(35.3)\end{array}$ & & $12 / 128(9.4)$ & $\begin{array}{c}3 / 6 \\
(50.0)\end{array}$ & \\
\hline & & $3 / 15(20.0)$ & $\begin{array}{l}12 / 15 \\
(80.0)\end{array}$ & & $12 / 15(80.0)$ & $\begin{array}{l}3 / 15 \\
(20.0)\end{array}$ & \\
\hline \multirow[t]{2}{*}{$0.4-0.8^{*} 10^{\wedge} 9 / \mathrm{L}(\mathrm{n}=46)$} & $46 / 134(34.3)$ & $\begin{array}{l}30 / 100 \\
(30.0)\end{array}$ & $\begin{array}{l}16 / 34 \\
(47.1)\end{array}$ & & $\begin{array}{l}44 / 128 \\
(34.4)\end{array}$ & $\begin{array}{c}2 / 6 \\
(33.3)\end{array}$ & \\
\hline & & $\begin{array}{l}30 / 46 \\
(65.2)\end{array}$ & $\begin{array}{l}16 / 46 \\
(34.8)\end{array}$ & & $44 / 46(95.7)$ & $\begin{array}{l}2 / 46 \\
(4.3)\end{array}$ & \\
\hline \multirow[t]{2}{*}{$\square 0.8^{*} 10^{\wedge} 9 / \mathrm{L}(\mathrm{n}=73)$} & $73 / 134$ (54.5) & $\begin{array}{l}67 / 100 \\
(67.0)\end{array}$ & $6 / 34(17.6)$ & & $\begin{array}{l}72 / 128 \\
(56.3)\end{array}$ & $\begin{array}{c}1 / 6 \\
(16.7)\end{array}$ & \\
\hline & & $\begin{array}{l}67 / 73 \\
(91.8)\end{array}$ & $6 / 73(8.2)$ & & $72 / 73(98.6)$ & $\begin{array}{l}1 / 73 \\
(1.4)\end{array}$ & \\
\hline $\begin{array}{l}\text { Highest C-reactive protein } \\
\text { hospitalization-No., } \%\end{array}$ & level during & & & $\square 0.001$ & & & $\square 0.001$ \\
\hline \multirow[t]{3}{*}{$\square 20 \mathrm{mg} / \mathrm{L}(\mathrm{n}=57)$} & $57 / 134(42.5)$ & $\begin{array}{c}56 / 100 \\
(56.0)\end{array}$ & $1 / 34(2.9)$ & & $\begin{array}{l}57 / 128 \\
(44.5)\end{array}$ & $0 / 6(0.0)$ & \\
\hline & & $56 / 57$ & $1 / 57(1.8)$ & & $57 / 57$ & $0 / 57$ & \\
\hline & & Page $11 / 30$ & & & & & \\
\hline
\end{tabular}




\begin{tabular}{|c|c|c|c|c|c|c|c|}
\hline & & $(98.2)$ & & & $(100.0)$ & $(0.0)$ & \\
\hline \multirow[t]{2}{*}{$20-90 \mathrm{mg} / \mathrm{L}(\mathrm{n}=38)$} & $38 / 134(28.4)$ & $\begin{array}{c}32 / 100 \\
(32.0)\end{array}$ & $6 / 34(17.6)$ & & $\begin{array}{c}38 / 128 \\
(29.7)\end{array}$ & $0 / 6(0.0)$ & \\
\hline & & $\begin{array}{l}32 / 38 \\
(84.2)\end{array}$ & $6 / 38(15.8)$ & & $\begin{array}{c}38 / 38 \\
(100.0)\end{array}$ & $\begin{array}{l}0 / 38 \\
(0.0)\end{array}$ & \\
\hline \multirow[t]{2}{*}{$90-150 \mathrm{mg} / \mathrm{L}(\mathrm{n}=16)$} & 16/134 (11.9) & $9 / 100(9.0)$ & $7 / 34(20.6)$ & & $\begin{array}{c}16 / 128 \\
(12.5)\end{array}$ & $0 / 6(0.0)$ & \\
\hline & & $9 / 16(56.3)$ & $7 / 16(43.8)$ & & $\begin{array}{c}16 / 16 \\
(100.0)\end{array}$ & $\begin{array}{l}0 / 16 \\
(0.0)\end{array}$ & \\
\hline \multirow[t]{2}{*}{$\square 150 \mathrm{mg} / \mathrm{L}(\mathrm{n}=23)$} & $23 / 134(17.2)$ & $3 / 100(3.0)$ & $\begin{array}{l}20 / 34 \\
(58.8)\end{array}$ & & $\begin{array}{c}17 / 128 \\
(13.3)\end{array}$ & $\begin{array}{c}6 / 6 \\
(100.0)\end{array}$ & \\
\hline & & $3 / 23(13.0)$ & $\begin{array}{l}20 / 23 \\
(87.0)\end{array}$ & & $17 / 23(73.9)$ & $\begin{array}{c}6 / 23 \\
(26.1)\end{array}$ & \\
\hline \multicolumn{2}{|c|}{ est D-dimer level during hospitalization-No., \% } & & & {$[0.001$} & & & $\square 0.001$ \\
\hline \multirow[t]{2}{*}{$\leq 1 \mathrm{mg} / \mathrm{L}(\mathrm{n}=98)$} & $98 / 134(73.1)$ & $\begin{array}{c}90 / 100 \\
(90.0)\end{array}$ & $8 / 34(23.5)$ & & $\begin{array}{c}98 / 128 \\
(76.6)\end{array}$ & $0 / 6(0.0)$ & \\
\hline & & $\begin{array}{l}90 / 98 \\
(91.8)\end{array}$ & $8 / 98(8.2)$ & & $\begin{array}{c}98 / 98 \\
(100.0)\end{array}$ & $\begin{array}{l}0 / 98 \\
(0.0)\end{array}$ & \\
\hline \multirow[t]{2}{*}{$\square 1 \mathrm{mg} / \mathrm{L}(\mathrm{n}=36)$} & $36 / 134(26.9)$ & $\begin{array}{c}10 / 100 \\
(10.0)\end{array}$ & $\begin{array}{l}26 / 34 \\
(76.5)\end{array}$ & & $\begin{array}{c}30 / 128 \\
(23.4)\end{array}$ & $\begin{array}{c}6 / 6 \\
(100.0)\end{array}$ & \\
\hline & & $\begin{array}{l}10 / 36 \\
(27.8)\end{array}$ & $\begin{array}{l}26 / 36 \\
(72.2)\end{array}$ & & $30 / 36(83.3)$ & $\begin{array}{c}6 / 36 \\
(16.7)\end{array}$ & \\
\hline
\end{tabular}

\begin{tabular}{|c|c|c|c|}
\hline \multicolumn{4}{|l|}{$\begin{array}{l}\text { Highest a } \\
\text { No., } \%\end{array}$} \\
\hline \multirow[t]{2}{*}{$\square 40 \mathrm{U} / \mathrm{L}(\mathrm{n}=77)$} & $77 / 134(57.5)$ & $\begin{array}{c}71 / 100 \\
(71.0)\end{array}$ & 6/34 (17.6) \\
\hline & & $\begin{array}{l}71 / 77 \\
(92.2)\end{array}$ & $6 / 77(7.8)$ \\
\hline \multirow[t]{2}{*}{$40-80 \mathrm{U} / \mathrm{L}(\mathrm{n}=32)$} & $32 / 134$ (23.9) & $\begin{array}{c}18 / 100 \\
(18.0)\end{array}$ & $\begin{array}{l}14 / 34 \\
(41.2)\end{array}$ \\
\hline & & $\begin{array}{l}18 / 32 \\
(56.3)\end{array}$ & $\begin{array}{l}14 / 32 \\
(43.7)\end{array}$ \\
\hline \multirow[t]{2}{*}{$\square 80 \mathrm{U} / \mathrm{L}(\mathrm{n}=25)$} & $25 / 134$ (18.7) & $\begin{array}{c}11 / 100 \\
(11.0)\end{array}$ & $\begin{array}{l}14 / 34 \\
(41.2)\end{array}$ \\
\hline & & $\begin{array}{l}11 / 25 \\
(44.0)\end{array}$ & $\begin{array}{l}14 / 25 \\
(56.0)\end{array}$ \\
\hline
\end{tabular}

$\square 0.001$

0.295

Highest aspartate aminotransferase activity during hospitalizationNo., \%

\begin{tabular}{|c|c|c|c|}
\hline \multirow[t]{2}{*}{$\square 40 \mathrm{U} / \mathrm{L}(\mathrm{n}=86)$} & $86 / 134(64.2)$ & $\begin{array}{c}77 / 100 \\
(77.0)\end{array}$ & 9/34 (26.5) \\
\hline & & $\begin{array}{l}77 / 86 \\
(89.5)\end{array}$ & 9/86 (10.5) \\
\hline \multirow[t]{2}{*}{$40-80 \mathrm{U} / \mathrm{L}(\mathrm{n}=37)$} & $37 / 134(27.6)$ & $\begin{array}{c}19 / 100 \\
(19.0)\end{array}$ & $\begin{array}{l}18 / 34 \\
(52.9)\end{array}$ \\
\hline & & $\begin{array}{l}19 / 37 \\
(51.4)\end{array}$ & $\begin{array}{l}18 / 37 \\
(48.6)\end{array}$ \\
\hline \multirow[t]{3}{*}{$\square 80 \mathrm{U} / \mathrm{L}(\mathrm{n}=11)$} & $11 / 134$ (8.2) & $4 / 100(4.0)$ & $7 / 34(20.6)$ \\
\hline & & $4 / 11(36.4)$ & 7/11 (63.6) \\
\hline & & Page $12 / 3$ & \\
\hline
\end{tabular}

$\square 0.001$

0.003

$\begin{array}{cc}86 / 128 & 0 / 6(0.0) \\ (67.2) & \\ 86 / 86 & 0 / 86 \\ (100.0) & (0.0) \\ 32 / 128 & 5 / 6 \\ (25.0) & (83.3) \\ 32 / 37(86.5) & 5 / 37 \\ & (13.5) \\ 10 / 128(7.8) & 1 / 6 \\ & (16.7) \\ 10 / 11(90.9) & 1 / 11\end{array}$


Highest Creatinine level during hospitalization-No., $\%$

0.314

0.070

$\begin{array}{lccc}\leq 90 \mu \mathrm{mol} / \mathrm{L}(\mathrm{n}=110) & 110 / 134(82.1) & 84 / 100 & 26 / 34 \\ & & (84.0) & (76.5) \\ & & 84 / 110 & 26 / 110 \\ & & (76.4) & (23.6) \\ & & & \\ & & 16 / 100 & 8 / 34(23.5) \\ & 24 / 134(17.9) & (16.0) & \\ & & 16 / 24 & 8 / 24(33.3)\end{array}$

Highest $\alpha$ - hydroxybutyrate dehydrogenase activity during hospitalization-No., \%

$\square 0.001$

$\begin{array}{cc}107 / 128 & 3 / 6 \\ (83.6) & (50.0) \\ 107 / 110 & 3 / 110 \\ (97.3) & (2.7) \\ 21 / 128 & 3 / 6 \\ (16.4) & (50.0) \\ 21 / 24(87.5) & 3 / 24 \\ & (12.5)\end{array}$

$\begin{array}{cccc}\square 183 \mathrm{U} / \mathrm{L}(\mathrm{n}=30) & 30 / 134(22.4) & \begin{array}{c}30 / 100 \\ (30.0)\end{array} & 0 / 34(0.0) \\ & & 30 / 30 & 0 / 30(0.0) \\ 183-360 \mathrm{U} / \mathrm{L}(\mathrm{n}=64) & 53 / 100 & 11 / 34 \\ & & (53.0) & (32.4) \\ & & 53 / 64 & 11 / 64 \\ 360-540 \mathrm{U} / \mathrm{L}(\mathrm{n}=31) & (82.8) & (17.2) \\ & & 17 / 100 & 14 / 34 \\ & & (17.0) & (41.2) \\ & & 17 / 31 & 14 / 31 \\ & & (54.8) & (45.2) \\ & & 0 / 100(0.0) & 9 / 34(26.5) \\ & & & \\ & & & \\ & & 0 / 19(0.0) & 9 / 9(100.0)\end{array}$

Highest lactate dehydrogenase activity during hospitalization-No., \%

$\square 0.001$

$\begin{array}{cc}30 / 128 & 0 / 6(0.0) \\ (23.4) & \\ 30 / 30 & 0 / 30 \\ (100.0) & (0.0) \\ 64 / 128 & 0 / 6(0.0) \\ (50.0) & \\ 64 / 64 & 0 / 64 \\ (100.0) & (0.0) \\ 31 / 128 & 0 / 6(0.0) \\ (24.2) & \\ 31 / 31 & 0 / 31 \\ (100.0) & (0.0) \\ 3 / 128(2.3) & 6 / 6 \\ & (100.0) \\ 3 / 9(33.3) & 6 / 9 \\ & (66.7)\end{array}$

[0.001

$$
\square 245 \mathrm{U} / \mathrm{L}(\mathrm{n}=52)
$$

$52 / 134(38.8)$

$51 / 100$

245-480 U/L $(\mathrm{n}=64)$

$\square 720 \mathrm{U} / \mathrm{L}(\mathrm{n}=6)$

480-720 U/L $(n=12)$

$12 / 134(9.0)$

6/134 (4.5)
(51.0)

$51 / 52$

(98.1)

$0 / 100(0.0) \quad 6 / 34(17.6)$

$0 / 6(0.0)$

1/34 (2.9)

$1 / 52(1.9)$

$20 / 34$

(58.8)

$20 / 64$

(31.2)

(68.8)

$5 / 12(41.7) \quad 7 / 12(58.3)$

$52 / 128 \quad 0 / 6(0.0)$

(40.6)

$52 / 52$

(100.0)

$0 / 52$

$64 / 128 \quad 0 / 6(0.0)$

(50.0)

$64 / 64 \quad 0 / 64$

(100.0) (0.0)

$11 / 128(8.6) \quad 1 / 6$

(16.7)

$11 / 12(91.7) \quad 1 / 12$

(8.3)

$1 / 128(0.8) \quad 5 / 6$ (83.3)

$1 / 6(16.7) \quad 5 / 6$ (83.3)

0.032

$\square 0.001$ 


\begin{tabular}{|c|c|c|c|c|c|}
\hline & & $\begin{array}{c}81 / 101 \\
(80.2)\end{array}$ & $\begin{array}{c}20 / 101 \\
(19.8)\end{array}$ & $\begin{array}{c}99 / 101 \\
(98.0)\end{array}$ & $\begin{array}{c}2 / 101 \\
(2.0)\end{array}$ \\
\hline $00-400 U / L(n=13)$ & $13 / 134(9.7)$ & $8 / 100(8.0)$ & $5 / 34(14.7)$ & $12 / 128(9.4)$ & $\begin{array}{c}1 / 6 \\
(16.7)\end{array}$ \\
\hline & & $8 / 13(61.5)$ & $5 / 13(38.5)$ & $12 / 13(92.3)$ & $\begin{array}{l}1 / 13 \\
(7.7)\end{array}$ \\
\hline & & $\begin{array}{l}11 / 20 \\
(55.0)\end{array}$ & $9 / 20(45.0)$ & $17 / 20(85.0)$ & $\begin{array}{c}3 / 20 \\
(15.0)\end{array}$ \\
\hline
\end{tabular}

P1 values denote comparison between nonsevere and severe cases

P2 values denote comparison between survivors and nonsurvivors

Table 4. Laboratory findings for 134 patients with COVID-19 


\begin{tabular}{|c|c|c|c|c|c|c|c|}
\hline \multirow[b]{2}{*}{ Laboratory findings } & \multirow{2}{*}{$\begin{array}{l}\text { All patients } \\
\qquad(\mathrm{n}=134)\end{array}$} & \multicolumn{2}{|c|}{ Disease severity } & \multicolumn{3}{|c|}{ Outcome } & \multirow[b]{2}{*}{ P2 } \\
\hline & & $\begin{array}{c}\text { No-severe } \\
(n=100)\end{array}$ & $\begin{array}{l}\text { Severe } \\
(n=34)\end{array}$ & P1 & $\begin{array}{l}\text { Survival } \\
(\mathrm{n}=128)\end{array}$ & $\begin{array}{l}\text { Death } \\
(n=6)\end{array}$ & \\
\hline \multicolumn{2}{|c|}{ Blood leukocyte count on admission, No., \% } & & & $\square 0.001$ & & & 0.001 \\
\hline \multirow[t]{2}{*}{$>10^{*} 10^{\wedge} 9 / \mathrm{L}(\mathrm{n}=11)$} & $11 / 134(8.2)$ & $1 / 100(1.0)$ & 10/34 (14.7) & & $8 / 128(6.3)$ & $3 / 6(50.0)$ & \\
\hline & & $1 / 11(9.1)$ & $10 / 11(90.9)$ & & $8 / 11(72.7)$ & $3 / 11(27.3)$ & \\
\hline \multirow[t]{2}{*}{$4-10^{*} 10^{\wedge} 9 / \mathrm{L}(\mathrm{n}=94)$} & $94 / 134(70.1)$ & $75 / 100(75.0)$ & 19/34 (55.9) & & $91 / 128(71.1)$ & $3 / 6(50.0)$ & \\
\hline & & $75 / 94(79.8)$ & $19 / 94(20.2)$ & & $91 / 94(96.8)$ & $3 / 94(3.2)$ & \\
\hline \multirow[t]{2}{*}{$\square 4^{*} 10^{\wedge} 9 / \mathrm{L}(\mathrm{n}=29)$} & $29 / 134(21.6)$ & $24 / 100(24.0)$ & $5 / 34(29.4)$ & & $29 / 128(22.7)$ & $0 / 6(0.0)$ & \\
\hline & & $24 / 29(82.8)$ & $5 / 29(17.2)$ & & $29 / 29(100.0)$ & $0 / 29(0.0)$ & \\
\hline \multicolumn{2}{|c|}{ Neutrophil count on admission-No., \% } & & & $\square 0.001$ & & & $\square 0.001$ \\
\hline \multirow[t]{2}{*}{$>7 * 10^{\wedge} 9 / \mathrm{L}(\mathrm{n}=18)$} & $18 / 134(13.4)$ & $2 / 100(2.0)$ & $16 / 34(47.1)$ & & $14 / 128(10.9)$ & $4 / 6(66.7)$ & \\
\hline & & $2 / 18(11.1)$ & $16 / 18(88.9)$ & & $14 / 18(77.8)$ & $4 / 18(22.2)$ & \\
\hline \multirow[t]{2}{*}{$2-7 * 10^{\wedge} 9 / \mathrm{L}(\mathrm{n}=94)$} & $94 / 134(70.1)$ & $79 / 100(79.0)$ & $15 / 34(44.1)$ & & $92 / 128(71.9)$ & $2 / 6(33.3)$ & \\
\hline & & $79 / 94(84.0)$ & $15 / 94(16.0)$ & & $92 / 94(97.9)$ & $2 / 94(2.1)$ & \\
\hline \multirow[t]{2}{*}{ प $2 * 10^{*} 9 / \mathrm{L}(\mathrm{n}=22)$} & $22 / 134(16.4)$ & $19 / 100(19.0)$ & $3 / 34(8.8)$ & & $22 / 128(17.2)$ & $0 / 6(0.0)$ & \\
\hline & & $19 / 22(86.4)$ & $3 / 22(13.6)$ & & $22 / 22(100.0)$ & $0 / 22(0.0)$ & \\
\hline \multicolumn{2}{|c|}{ Lymphocyte count on admission-No., \% } & & & $\square 0.001$ & & & $\square 0.001$ \\
\hline \multirow[t]{2}{*}{$0.4^{*} 0^{\wedge} 9 / \mathrm{L}(\mathrm{n}=4)$} & $4 / 134(3.0)$ & $0 / 100(0.0)$ & $4 / 34(11.8)$ & & $2 / 128(1.6)$ & $2 / 6(33.3)$ & \\
\hline & & $0 / 4(0.0)$ & $4 / 4(100.0)$ & & $2 / 4(50.0)$ & $2 / 4(50.0)$ & \\
\hline \multirow[t]{2}{*}{$0.4-0.8^{*} 10^{\wedge} 9 / \mathrm{L}(\mathrm{n}=36)$} & $36 / 134(26.9)$ & $23 / 100(23.0)$ & 13/34 (38.2) & & $35 / 128(27.3)$ & $1 / 6(16.7)$ & \\
\hline & & $23 / 36(63.9)$ & 13/36 (36.1) & & $35 / 36(97.2)$ & $1 / 36(2.8)$ & \\
\hline \multirow[t]{2}{*}{$\square 0.8^{*} 10^{\wedge} 9 / \mathrm{L}(\mathrm{n}=94)$} & $94 / 134(70.1)$ & $77 / 100(77.0)$ & $17 / 34(50.0)$ & & $91 / 128(71.1)$ & $3 / 6(50.0)$ & \\
\hline & & $77 / 94(81.9)$ & 17/94 (18.1) & & $91 / 94(96.8)$ & $3 / 94(3.2)$ & \\
\hline \multicolumn{2}{|c|}{ C-reactive protein level on admission-No., \% } & & & $\square 0.001$ & & & 0.009 \\
\hline \multirow[t]{2}{*}{$\square 20 \mathrm{mg} / \mathrm{L}(\mathrm{n}=68)$} & $68 / 134(50.7)$ & $65 / 100(65.0)$ & $3 / 34(8.8)$ & & $68 / 128(53.1)$ & $0 / 6(0.0)$ & \\
\hline & & $65 / 68(95.6)$ & $3 / 68(4.4)$ & & $68 / 68(100.0)$ & $0 / 68(0.0)$ & \\
\hline \multirow[t]{2}{*}{$20-90 \mathrm{mg} / \mathrm{L}(\mathrm{n}=42)$} & $42 / 134(31.3)$ & $28 / 100(28.0)$ & $14 / 34(41.2)$ & & $40 / 128(31.3)$ & $2 / 6(33.3)$ & \\
\hline & & $28 / 42(66.7)$ & $14 / 42(33.3)$ & & $40 / 42(95.2)$ & $2 / 42(4.8)$ & \\
\hline \multirow[t]{2}{*}{$90-150 \mathrm{mg} / \mathrm{L}(\mathrm{n}=11)$} & $11 / 134(8.2)$ & $4 / 100(4.0)$ & $7 / 34(20.6)$ & & $9 / 128(7.0)$ & $2 / 6(33.3)$ & \\
\hline & & $4 / 11(36.4)$ & $7 / 11(63.6)$ & & 9/11 (81.8) & $2 / 11(18.2)$ & \\
\hline \multirow[t]{2}{*}{$\square 150 \mathrm{mg} / \mathrm{L}(\mathrm{n}=13)$} & $13 / 134(9.7)$ & $3 / 100(3.0)$ & $10 / 34(29.4)$ & & $11 / 128(8.6)$ & $2 / 6(33.3)$ & \\
\hline & & $3 / 13(23.1)$ & 10/13 (76.9) & & $11 / 13(84.6)$ & $2 / 13(15.4)$ & \\
\hline \multicolumn{2}{|c|}{ D-dimer level on admission-No., \% } & & & $\square 0.001$ & & & 0.037 \\
\hline \multirow[t]{2}{*}{$\leq 1 \mathrm{mg} / \mathrm{L}(\mathrm{n}=115)$} & $115 / 134(85.8)$ & $95 / 100(95.0)$ & 20/34 (58.8) & & $112 / 128(87.5)$ & $3 / 6(50.0)$ & \\
\hline & & $95 / 115(82.6)$ & $20 / 115(17.4)$ & & $112 / 115(97.4)$ & $3 / 115(2.6)$ & \\
\hline \multirow[t]{2}{*}{$\square 1 \mathrm{mg} / \mathrm{L}(\mathrm{n}=19)$} & $19 / 134(14.2)$ & $5 / 100(5.0)$ & $14 / 34(41.2)$ & & $16 / 128(12.5)$ & $3 / 6(50.0)$ & \\
\hline & & $5 / 19(26.3)$ & 14/19 (73.7) & & $16 / 19$ (84.2) & $3 / 19$ (15.8) & \\
\hline
\end{tabular}




\begin{tabular}{|c|c|c|c|c|c|c|c|}
\hline$\square 40 \mathrm{U} / \mathrm{L}(\mathrm{n}=97)$ & $97 / 134(72.4)$ & $81 / 100(81.0)$ & $16 / 34(47.1)$ & & $95 / 128(74.2)$ & $2 / 6(33.3)$ & \\
\hline & & $81 / 97$ (83.5) & $16 / 97(16.5)$ & & 95/97 (97.9) & $2 / 97(2.1)$ & \\
\hline $40-80 \mathrm{U} / \mathrm{L}(\mathrm{n}=33)$ & $33 / 134(24.6)$ & $18 / 100(18.0)$ & $15 / 34(44.1)$ & & $30 / 128(23.4)$ & $3 / 6(50.0)$ & \\
\hline & & $18 / 33(54.5)$ & $15 / 33(45.5)$ & & $30 / 33(90.9)$ & $3 / 33(9.1)$ & \\
\hline$\square 80 \mathrm{U} / \mathrm{L}(\mathrm{n}=4)$ & $4 / 134(3.0)$ & $1 / 100(1.0)$ & $3 / 34(8.8)$ & & $3 / 128(2.3)$ & $1 / 6(16.7)$ & \\
\hline & & $1 / 4(25.0)$ & $3 / 4(75.0)$ & & $3 / 4(75.0)$ & $1 / 4(25.0)$ & \\
\hline Aspartate aminotransfera & ctivity on admissi & on-No., \% & & {$[0.001$} & & & 0.004 \\
\hline$\square 40 \mathrm{U} / \mathrm{L}(\mathrm{n}=99)$ & 99/134 (73.9) & $83 / 100(83.0)$ & $16 / 34(47.1)$ & & $98 / 128(76.6)$ & $1 / 6(16.7)$ & \\
\hline & & 83/99 (83.8) & $16 / 99(16.2)$ & & 98/99 (99.0) & $1 / 99(1.0)$ & \\
\hline $40-80 \mathrm{U} / \mathrm{L}(\mathrm{n}=30)$ & $30 / 134(22.4)$ & $15 / 100(15.0)$ & $15 / 34(44.1)$ & & $26 / 128(20.3)$ & $4 / 6(66.7)$ & \\
\hline & & $15 / 30(50.0)$ & $15 / 30(50.0)$ & & $26 / 30(86.7)$ & $4 / 30(3.3)$ & \\
\hline$\square 80 \mathrm{U} / \mathrm{L}(\mathrm{n}=5)$ & $5 / 134(3.7)$ & $2 / 100(2.0)$ & $3 / 34(8.8)$ & & $4 / 128(3.1)$ & $1 / 6(16.7)$ & \\
\hline & & $2 / 5(40.0)$ & $3 / 5(60.0)$ & & $4 / 5(80.0)$ & $1 / 5(20.0)$ & \\
\hline Creatinine level on admis & -No., \% & & & 1.000 & & & 1.000 \\
\hline$\leq 90 \mu \mathrm{mol} / \mathrm{L}(\mathrm{n}=130)$ & $130 / 134(97.0)$ & $97 / 100(97.0)$ & $33 / 34(97.1)$ & & $124 / 128(96.9)$ & 6/6 (100.0) & \\
\hline & & $97 / 130(74.6)$ & $33 / 130(25.4)$ & & $124 / 130(95.4)$ & $6 / 130(4.6)$ & \\
\hline$\square 90 \mu \mathrm{mol} / \mathrm{L}(\mathrm{n}=4)$ & $4 / 134(3.0)$ & $3 / 100(3.0)$ & $1 / 34(2.9)$ & & $4 / 128(3.1)$ & $0 / 6(0.0)$ & \\
\hline & & $3 / 4(75.0)$ & $1 / 4(25.0)$ & & $4 / 4(100.0)$ & $0 / 4(0.0)$ & \\
\hline$\alpha$ - hydroxybutyrate dehyc & nase activity on & Idmission-No., \% & & 00.001 & & & {$[0.001$} \\
\hline$\square 183 \mathrm{U} / \mathrm{L}(\mathrm{n}=40)$ & 40/134 (29.9) & $40 / 100(40.0)$ & $0 / 34(0.0)$ & & $40 / 128(31.3)$ & $0 / 6(0.0)$ & \\
\hline & & $40 / 40(100.0)$ & $0 / 40(0.0)$ & & $40 / 40(100.0)$ & $0 / 40(0.0)$ & \\
\hline $183-360 \mathrm{U} / \mathrm{L}(\mathrm{n}=67)$ & $67 / 134(50.0)$ & $49 / 100(49.0)$ & $18 / 34(52.9)$ & & $65 / 128(50.8)$ & $2 / 6(33.3)$ & \\
\hline & & $49 / 67(73.1)$ & $18 / 67(26.9)$ & & $65 / 67(97.0)$ & $2 / 67(3.0)$ & \\
\hline $360-540 \mathrm{U} / \mathrm{L}(\mathrm{n}=25)$ & 25/134 (18.7) & $11 / 100(11.0)$ & $14 / 34(41.2)$ & & $23 / 128(18.0)$ & $2 / 6(33.3)$ & \\
\hline & & $11 / 25(44.0)$ & $14 / 25(56.0)$ & & $23 / 25(92.0)$ & $2 / 25(8.0)$ & \\
\hline$\square 540 \mathrm{U} / \mathrm{L}(\mathrm{n}=2)$ & $2 / 134(1.5)$ & $0 / 100(0.0)$ & $2 / 34(5.9)$ & & $0 / 128(0.0)$ & $2 / 6(33.3)$ & \\
\hline & & $0 / 2(0.0)$ & $2 / 2(100.0)$ & & $0 / 2(0.0)$ & $2 / 2(100.0)$ & \\
\hline Lactate dehydrogenase a & y on admission- $N$ & $0 ., \%$ & & 00.001 & & & $\square 0.001$ \\
\hline$\square 245 \mathrm{U} / \mathrm{L}(\mathrm{n}=64)$ & $64 / 134(47.8)$ & $62 / 100(62.0)$ & $2 / 34(5.9)$ & & $63 / 128(49.2)$ & $1 / 6(16.7)$ & \\
\hline & & $62 / 64(96.9)$ & $2 / 64(3.1)$ & & 63/64 (98.4) & $1 / 64(1.6)$ & \\
\hline $245-480 \mathrm{U} / \mathrm{L}(\mathrm{n}=57)$ & $51 / 134(42.5)$ & $34 / 100(34.0)$ & $23 / 34(67.6)$ & & $56 / 128(43.8)$ & $1 / 6(16.7)$ & \\
\hline & & $34 / 57(59.6)$ & $23 / 57(40.4)$ & & $56 / 57(98.2)$ & $1 / 57(1.8)$ & \\
\hline $480-720 \mathrm{U} / \mathrm{L}(\mathrm{n}=11)$ & $11 / 134(8.2)$ & $4 / 100(4.0)$ & $7 / 34(20.6)$ & & $9 / 128(7.0)$ & $2 / 6(33.3)$ & \\
\hline & & $4 / 11(36.4)$ & $7 / 11(63.6)$ & & 9/11 (81.8) & $2 / 11(18.2)$ & \\
\hline$\square 720 \mathrm{U} / \mathrm{L}(\mathrm{n}=2)$ & $2 / 134(1.5)$ & $0 / 100(0.0)$ & $2 / 34(5.9)$ & & $0 / 128(0.0)$ & $2 / 6(33.3)$ & \\
\hline & & $0 / 2(0.0)$ & $2 / 2(100.0)$ & & $0 / 2(0.0)$ & $2 / 2(100.0)$ & \\
\hline Creatine kinase activity o & mission-No., \% & & & 0.020 & & & 0.010 \\
\hline$\square 200 \mathrm{U} / \mathrm{L}(\mathrm{n}=108)$ & $108 / 134(80.6)$ & $86 / 100(86.0)$ & $22 / 34(64.7)$ & & 106/128 (82.8) & $2 / 6(33.3)$ & \\
\hline
\end{tabular}




\begin{tabular}{|c|c|c|c|c|c|}
\hline & & $86 / 108$ (79.6) & $22 / 108(20.4)$ & $106 / 108(98.1)$ & $2 / 108$ (1.9) \\
\hline \multirow[t]{2}{*}{$200-400 U / L(n=8)$} & $8 / 134(6.0)$ & $5 / 100(5.0)$ & $3 / 34(8.8)$ & $7 / 128(5.5)$ & 1/6 (16.7) \\
\hline & & $5 / 8(62.5)$ & $3 / 8(37.5)$ & $7 / 8(87.5)$ & $1 / 8(12.5)$ \\
\hline \multirow[t]{2}{*}{$\square 400 \mathrm{U} / \mathrm{L}(\mathrm{n}=18)$} & $18 / 134(13.4)$ & $9 / 100(9.0)$ & $9 / 34(26.5)$ & 15/128 (11.7) & $3 / 6(50.0)$ \\
\hline & & $9 / 18(50.0)$ & $9 / 18(50.0)$ & 15/18 (83.3) & $3 / 18$ (16.7) \\
\hline
\end{tabular}

P1 values denote comparison between nonsevere and severe cases

P2 values denote comparison between survivors and nonsurvivors

\subsubsection{Blood leukocyte count}

\subsubsection{Highest blood leukocyte count during hospitalization}

As shown in Table 3, we divided patients into three groups according to their highest blood leukocyte count during hospitalization.

The results were as follows: for the $>10^{\star} 10^{\wedge} 9 / \mathrm{L}$ group, $92.6 \%$ of the patients had severe cases, and $22.2 \%$ of them died; for the $4-10^{\star} 10^{\wedge} 9 / \mathrm{L}$ group, $7.5 \%$ of the patients had severe cases, and none of them died; for the $\varangle 4^{*} 10^{\wedge} 9 / \mathrm{L}$ group, $14.3 \%$ of the patients had severe cases, and none of them died. (P1区0.001, P2囚 0.001)

A blood leukocyte count of $>10^{\star} 10^{\wedge} 9 / \mathrm{L}$ during hospitalization was strongly related to severe COVID-19 and an outcome of death.

\subsubsection{Blood leukocyte count on admission}

Table 4 divides patients into three groups according to their blood leukocyte count on admission.

The data for severe COVID-19 and death were as follows: for the $>10^{\star} 10^{\wedge} 9 / \mathrm{L}$ group, $90.9 \%$ and $27.3 \%$; for the $4-10^{*} 10^{\wedge} 9 / \mathrm{L}$ group, $20.2 \%$ and $3.2 \%$; for the $<4 * 10^{\wedge} 9 / \mathrm{L}$ group, $17.2 \%$ and none, respectively. $(\mathrm{P} 1<0.001, \mathrm{P} 2<0.001)$

A blood leukocyte count of $>10^{\star} 10^{\wedge} 9 / \mathrm{L}$ on admission was strongly associated with severe disease and death.

\subsubsection{Neutrophils}

3.3.2.1 Highest neutrophil count during hospitalization

As shown in Table 3, we grouped patients into three groups according to their highest neutrophil count during hospitalization. 
The data for severe COVID-19 and death were as follows: for the $>7 \star 10^{\wedge} 9 / \mathrm{L}$ group, $83.9 \%$ and $19.4 \%$; for the $2-7 * 10^{\wedge} 9 / \mathrm{L}$ group, $8.5 \%$ and none; for the $<2 * 10^{\wedge} 9 / \mathrm{L}$ group, none and none, respectively. $(\mathrm{P} 1<0.001$, $\mathrm{P} 2<0.001)$

A blood neutrophil count $>7 * 10^{\wedge} 9 / \mathrm{L}$ during hospitalization was strongly related to severe COVID-19 and death.

\subsubsection{Neutrophil count on admission}

As shown in Table 4, we grouped patients into three groups according to their neutrophil count on admission.

The data for severe COVID-19 and death were as follows: for the $>7 * 10^{\wedge} 9 / \mathrm{L}$ group, $88.9 \%$ and $22.2 \%$; for the $2-7 * 10^{\wedge} 9 / \mathrm{L}$ group, $16.0 \%$ and $2.1 \%$; for the $<2 * 10^{\wedge} 9 / \mathrm{L}$ group, $13.6 \%$ and none, respectively. $(\mathrm{P} 1<0.001$, $\mathrm{P} 2<0.001)$

A blood neutrophil count $>7 * 10^{\wedge} 9 / \mathrm{L}$ on admission was strongly related to severe COVID-19 and an outcome of death.

\subsubsection{Lymphocytes}

\subsubsection{Lowest lymphocyte count during hospitalization}

We also divided patients into three groups according to their lowest lymphocyte count during hospitalization (Table 3).

The results were as follows: for the $<0.4^{\star} 10^{\wedge} 9 / \mathrm{L}$ group, $80.0 \%$ of the patients had severe disease, and $20.0 \%$ died; for the $0.4-0.8^{*} 10^{\wedge} 9 / \mathrm{L}$ group, $34.8 \%$ of the patients had severe disease, and $4.3 \%$ died; For the $>0.8^{*} 10^{\wedge} 9 / \mathrm{L}$ group, $8.2 \%$ of the patients had severe disease, and $1.4 \%$ died. $(\mathrm{P} 1<0.001, \mathrm{P} 2<0.001)$

A lower lymphocyte count during hospitalization was strongly linked to severe COVID-19 and an outcome of death.

\subsubsection{Lymphocyte count on admission}

As shown in Table 4, we grouped patients according to their lymphocyte count on admission.

The data for severe COVID-19 and death were as follows: for the $<0.4 * 10^{\wedge} 9 / \mathrm{L}$ group, all and $50.0 \%$; for the $0.4-0.8^{*} 10^{\wedge} 9 / \mathrm{L}$ group, $36.1 \%$ and $2.8 \%$; for the $>0.8^{*} 10^{\wedge} 9 / \mathrm{L}$ group, $18.1 \%$ and $3.2 \%$, respectively. $(\mathrm{P} 1<0.001, \mathrm{P} 2 \bigotimes 0.001)$

A lower lymphocyte count on admission was strongly related to severe COVID-19 and death.

\subsubsection{C-reactive protein level}


3.3.4.1 Highest C-reactive protein level during hospitalization

As presented in Table 3, we grouped patients into four groups according to their highest C-reactive protein level during hospitalization.

The data for severe COVID-19 and death were as follows: for the $<20 \mathrm{mg} / \mathrm{L}$ group, $1.8 \%$ and none; for the 20-90 mg/L group, $15.8 \%$ and none; for the $90-150 \mathrm{mg} / \mathrm{L}$ group, $43.8 \%$ and none; for the $>150 \mathrm{mg} / \mathrm{L}$ group, $87.0 \%$ and $26.1 \%$, respectively. ( $P 1<0.001, \mathrm{P} 2<0.001)$

Higher C-reactive protein levels during hospitalization were strongly related to severe COVID-19 and an outcome of death.

\subsubsection{C-reactive protein level on admission}

We also classified patients into four groups according to their C-reactive protein level on admission (Table 4).

The data for severe COVID-19 and death were as follows: for the $<20 \mathrm{mg} / \mathrm{L}$ group, $4.4 \%$ and none; for the $20-90 \mathrm{mg} / \mathrm{L}$ group, $33.3 \%$ and $4.8 \%$; for the $90-150 \mathrm{mg} / \mathrm{L}$ group, $63.6 \%$ and $18.2 \%$; for the $>150 \mathrm{mg} / \mathrm{L}$ group, $76.9 \%$ and $15.4 \%$, respectively. (P1区0.001, P2=0.009)

Higher C-reactive protein levels on admission were strongly associated with severe COVID-19.

\subsubsection{D-dimer level}

\subsubsection{Highest D-dimer level during hospitalization}

As shown in Table 3, we grouped patients according to their highest D-dimer level during hospitalization.

For the $\leq 1 \mathrm{mg} / \mathrm{L}$ group, $8.2 \%$ of the patients had severe cases, and none of the patients died. For the $>1$ $\mathrm{mg} / \mathrm{L}$ group, $72.2 \%$ of the patients had severe cases, and $16.7 \%$ died. $(\mathrm{P} 1<0.001, \mathrm{P} 2<0.001)$

A higher D-dimer level was strongly linked to severe COVID-19 and an outcome of death.

\subsubsection{D-dimer level on admission}

We also grouped patients into two groups according to their D-dimer level on admission (Table 4).

For the $\leq 1 \mathrm{mg} / \mathrm{L}$ group, $17.4 \%$ of the patients had severe illness, and $2.6 \%$ of died. For the $>1 \mathrm{mg} / \mathrm{L}$ group, $73.7 \%$ had severe disease, and $15.8 \%$ of the patients died. (P1<0.001, P2 $=0.037)$

A higher D-dimer level on admission was strongly related to severe COVID-19 and an outcome of death.

\subsubsection{Alanine aminotransferase activity}

3.3.6.1 Highest alanine aminotransferase activity during hospitalization 
As shown in Table 3, we grouped patients according to their highest alanine aminotransferase activity during hospitalization.

For the $<40 \mathrm{U} / \mathrm{L}$ group, $7.8 \%$ of the patients had severe cases, and $2.6 \%$ of them died. For the $40-80 \mathrm{U} / \mathrm{L}$ group, $43.7 \%$ had severe cases, and $9.4 \%$ died. For the $>80 \mathrm{U} / \mathrm{L}$ group, $56.0 \%$ had severe cases, and $4.0 \%$ died. $(\mathrm{P} 1<0.001, \mathrm{P} 2=0.295)$

Higher alanine aminotransferase activity during hospitalization was associated with severe COVID-19.

\subsubsection{Alanine aminotransferase activity on admission}

Table 4 groups patients into three groups according to their alanine aminotransferase activity on admission.

The data for severe COVID-19 and death were as follows: for the $<40 \mathrm{U} / \mathrm{L}$ group, $16.5 \%$ and $2.1 \%$; for the 40-80 U/L group, $45.5 \%$ and $9.1 \%$; For the $>80 \mathrm{U} / \mathrm{L}$ group, $75.0 \%$ and $25.0 \%$, respectively. $(\mathrm{P} 1<0.001$, $\mathrm{P} 2=0.032)$

Higher alanine aminotransferase activity on admission was related to severe COVID-19 and an outcome of death.

\subsubsection{Aspartate aminotransferase activity}

\subsubsection{Highest aspartate aminotransferase activity during hospitalization}

As shown in Table 3, we grouped patients into three groups according to their highest aspartate aminotransferase activity during hospitalization.

The data for severe COVID-19 and death were as follows; for the $<40 \mathrm{U} / \mathrm{L}$ group, $10.5 \%$ and none; for the 40-80 U/L group, $48.6 \%$ and $13.5 \%$; for the $>80 \mathrm{U} / \mathrm{L}$ group, $63.6 \%$ and $9.1 \%$, respectively. (P1<0.001, $\mathrm{P} 2=0.003)$

Higher aspartate aminotransferase activity during hospitalization was also linked to severe COVID-19.

\subsubsection{Aspartate aminotransferase activity on admission}

As shown in Table 4, we grouped patients into three groups according to their aspartate aminotransferase activity on admission.

The data for severe COVID-19 and death were as follows: for the $<40 \mathrm{U} / \mathrm{L}$ group, $16.2 \%$ and $1.0 \%$; for the $40-80 \mathrm{U} / \mathrm{L}$ group, $50.0 \%$ and $3.3 \%$; for the $>80 \mathrm{U} / \mathrm{L}$ group, $60.0 \%$ and $20.0 \%$, respectively. (P1<0.001, $\mathrm{P} 2=0.004)$

Higher aspartate aminotransferase activity on admission was related to severe COVID-19 and an outcome of death. 


\subsubsection{Creatinine level}

\subsubsection{Highest creatinine level during hospitalization}

Patients were classified according to their highest creatinine level during hospitalization (Table 3).

The results were as follows: for the $\leq 90 \mu \mathrm{mol} / \mathrm{L}$ group, $23.6 \%$ of the patients had severe illness, and $2.7 \%$ died; for the $>90 \mu \mathrm{mol} / \mathrm{L}$ group, $33.3 \%$ had severe disease, and $12.5 \%$ died.

However, the $P$ values were greater than $0.05(P 1=0.314, P 2=0.07)$, and studies with larger samples under the same conditions are needed in the future.

\subsubsection{Creatinine level on admission}

As shown in Table 4, we grouped patients into according to their creatinine level on admission.

The results were as follows: for the $\leq 90 \mu \mathrm{mol} / \mathrm{L}$ group, $25.4 \%$ of the patients had severe cases, and $4.6 \%$ of the patients died; for the $>90 \mu \mathrm{mol} / \mathrm{L}$ group, $25.0 \%$ had severe cases, and none died.

As above, the $P$ values were greater than $0.05(P 1=1.000, P 2=1.000)$, and thus studies with larger samples under the same conditions are needed.

\subsection{9 a-Hydroxybutyrate dehydrogenase activity}

\subsubsection{Highest a-hydroxybutyrate dehydrogenase activity during hospitalization}

Table 3 groups patients according to their highest a-hydroxybutyrate dehydrogenase activity during hospitalization.

The data for severe COVID-19 and death were as follows: for the $<183 \mathrm{U} / \mathrm{L}$ group, none and none; for the 183-360 U/L group, $17.2 \%$ and none; for the $360-540 \mathrm{U} / \mathrm{L}$ group, $45.2 \%$ and none; for the $>540 \mathrm{U} / \mathrm{L}$ group, all and $66.7 \%$, respectively. (P1<0.001, $\mathrm{P} 2<0.001)$

Higher a-hydroxybutyrate dehydrogenase activity during hospitalization was related to severe COVID-19 and an outcome of death.

\subsubsection{0a-Hydroxybutyrate dehydrogenase activity on admission}

As shown in Table 4, we grouped patients into four groups according to their a-hydroxybutyrate dehydrogenase activity on admission.

The data for severe COVID-19 and death were as follows: for the $<183 \mathrm{U} / \mathrm{L}$ group, none and none; for the 183-360 U/L group, $26.9 \%$ and $3.0 \%$; for the $360-540 \mathrm{U} / \mathrm{L}$ group, $56.0 \%$ and $8.0 \%$; for the $>540 \mathrm{U} / \mathrm{L}$ group, all and all, respectively. (P1<0.001, $\mathrm{P} 2<0.001)$ 
Higher a-hydroxybutyrate dehydrogenase activity on admission was associated with severe COVID-19 and an outcome of death.

\subsubsection{Lactate dehydrogenase activity}

3.3.11.1 Highest lactate dehydrogenase activity during hospitalization

As indicated in Table 3, we grouped patients according to their highest lactate dehydrogenase activity during hospitalization.

The data for severe COVID-19 and death were as follows: for the $<245 \mathrm{U} / \mathrm{L}$ group, $1.9 \%$ and none; for the 245-480 U/L group, 31.2\% and none; for the $480-720 \mathrm{U} / \mathrm{L}$ group, $58.3 \%$ and $8.3 \%$; for the $>720 \mathrm{U} / \mathrm{L}$ group, all and $83.3 \%$, respectively. $(\mathrm{P} 1<0.001, \mathrm{P} 2<0.001)$

Higher lactate dehydrogenase activity during hospitalization was related to severe COVID-19 and an outcome of death.

\subsubsection{Lactate dehydrogenase activity on admission}

As shown in Table 4, we grouped patients into four groups according to their lactate dehydrogenase activity on admission.

The data for severe COVID-19 and death were as follows: for the $<245 \mathrm{U} / \mathrm{L}$ group, $3.1 \%$ and $1.6 \%$; for the 245-480 U/L group, $40.4 \%$ and $1.8 \%$; for the $480-720 \mathrm{U} / \mathrm{L}$ group, $63.6 \%$ and $18.2 \%$; for the $>720 \mathrm{U} / \mathrm{L}$ group, all and all, respectively. (P1<0.001, $\mathrm{P} 2<0.001)$

Higher lactate dehydrogenase activity on admission was found to be related to severe COVID-19 and death.

\subsubsection{Creatine kinase activity}

3.3.12.1 Highest creatine kinase activity during hospitalization

In Table 3, patients are classified into three groups according to their highest creatine kinase activity during hospitalization.

For the $<200 \mathrm{U} / \mathrm{L}$ group, $19.8 \%$ of the patients had severe cases, and $2.0 \%$ of the patients died. For the 200-400 U/L group, $38.5 \%$ of the patients had severe illness, and $7.7 \%$ died. For the $>400 \mathrm{U} / \mathrm{L}$ group, $45.0 \%$ of the patients developed severe disease, and $15.0 \%$ died. ( $P 1=0.032, P 2=0.031)$

Higher creatine kinase activity during hospitalization was related to severe COVID-19 and an outcome of death.

3.3.12.2 Creatine kinase activity on admission 
As shown in Table 4, we also grouped patients according to their creatine kinase activity on admission.

The data for severe COVID-19 and death were as follows: for the $<200 \mathrm{U} / \mathrm{L}$ group, $20.4 \%$ and $1.9 \%$; for the 200-400 U/L group, $37.5 \%$ and $12.5 \%$; for the $>400 \mathrm{U} / \mathrm{L}$ group, $50.0 \%$ and $16.7 \%$, respectively. (P1=0.020, $\mathrm{P} 2=0.010)$

Higher creatine kinase activity on admission was linked to severe COVID-19 and death.

\subsection{Administration of gamma globulin}

Administration of gamma globulin was not used as a conventional therapy method and depended on the wish of the patients and their relatives.

There were 34 severe cases that divided into two groups according to whether gamma globulin was used (Table 5).

Table 5. Therapy of 34 patients with severe COVID-19

\begin{tabular}{|c|c|c|c|c|}
\hline \multirow[b]{2}{*}{ Groups-No., \% } & \multirow{2}{*}{$\begin{array}{l}\text { All of the severe cases } \\
\qquad(\mathrm{n}=34)\end{array}$} & \multicolumn{3}{|c|}{ Outcomes } \\
\hline & & $\begin{array}{l}\text { Survival } \\
(\mathrm{n}=28)\end{array}$ & $\begin{array}{l}\text { Death } \\
(n=6)\end{array}$ & $\mathbf{P}$ \\
\hline Severe cases treated with gamma globulin $(n=16)$ & 16/34 (47.1) & $15 / 28(53.6)$ & $1 / 6(16.7)$ & 0.180 \\
\hline & & $15 / 16(93.8)$ & $1 / 16(6.2)$ & \\
\hline Severe cases treated without gamma globulin $(n=18)$ & $18 / 34(52.9)$ & $13 / 28(46.4)$ & $5 / 6(83.3)$ & \\
\hline & & $13 / 18(72.2)$ & $5 / 18(27.8)$ & \\
\hline
\end{tabular}

$\mathrm{P}$ values denote comparison between survivors and nonsurvivors

For severe cases treated with gamma globulin, $93.8 \%$ of the patients survived, and $6.2 \%$ died. Among severe cases not treated with gamma globulin, $72.2 \%$ survived, and $27.8 \%$ died.

It appears that administration of gamma globulin was helpful for reducing mortality among severe cases. However, the $P$ value was greater than $0.05(P=0.180)$, and future studies under the same conditions with larger samples are needed.

\section{Discussion}

\subsection{Underlying diseases and co-infection}

In total, $33.9 \%$ of all 186 patients had one or more severe underlying disease (i.e., chronic lung disease, chronic heart disease, chronic liver disease, chronic kidney disease) or were coinfected with other 
respiratory pathogens (i.e., bacteria, Chlamydia pneumoniae, Mycoplasma pneumoniae, adenovirus, or respiratory syncytial virus) associated with community-acquired pneumonia, as depicted in Table 1.

Compared with cases without comorbidity or coinfection, those with severe comorbidity or coinfection were more likely to progress to severe illness and had much higher mortality rates (Table 1).

Next, to more precisely assess the role of SARS-CoV-2 in COVID-19 without as little interference as possible, we excluded 52 patients with severe underlying disease or coinfection with other respiratory pathogens associated with community-acquired pneumonia. Ultimately, 134 patients with only COVID-19 were included, as illustrated in Figure 1.

\subsection{Clinical characteristics of 134 cases of COVID-19}

As shown in Table 2, fever, high temperature, cough, chest tightness, headaches, and fatigue were associated with severe COVID-19. Moreover, age older than 60 years, male sex, high temperature, chest tightness, and headaches were related to an outcome of death.

COVID-19 affects multiple systems of the human body, including the respiratory system, nervous system, muscular system, and digestive system, among others. In fact, it is reported that COVID-19 can cause damage to more than 8 systems. ${ }^{4}$ SARS-CoV-2 can infect a wide range of cell types by binding to ACE2 (angiotensin-converting enzyme 2), which is widely distributed in the human body. ${ }^{4}$

Males and the elderly had higher mortality rates than females and young people. Sex differences in response to inflammation have been documented and can be attributed, at least in part, to sex

hormones. ${ }^{5}$ Moreover, age-associated decreases in sex hormones, namely, estrogen and testosterone, can mediate proinflammatory increases in older adults, which may increase the risk of adverse outcomes in COVID-19. ${ }^{5}$

\subsection{Laboratory findings of 134 cases of COVID-19}

When assessing the relationship between potential risk factors and the severity of COVID-19, some studies have used the highest or lowest level of an indicator during hospitalization, whereas other studies have mainly utilized a fixed value on admission..$^{1-3}$ In ours study, we used both the fixed value on admission and the highest or lowest level during hospitalization of indicators to assess the relationship between potential risk factors and the severity of COVID-19.

According to Tables 3 and 4, high blood leukocyte and neutrophil counts, C-reactive protein and D-dimer levels, and alanine aminotransferase, aspartate aminotransferase, a-hydroxybutyrate dehydrogenase, lactate dehydrogenase and creatine kinase activities were related to severe COVID-19 and an outcome of death, as was a low lymphocyte count. Similar but not the same conclusions were reached when the evaluating the fixed value of an indicator on admission or the highest or lowest level during hospitalization. In our opinion, to identify risk factors related to severe COVID-19 and death, the highest or 
lowest level of an indicator during hospitalization might be more appropriate. This makes sense because the laboratory findings for patients on admission might be unrepresentative; indeed, laboratory findings may vary depending on the patient's health condition, and many cases that become severe during hospitalization might be nonsevere cases on admission. To address why similar conclusions were reached when using a fixed value on admission, some cases were already severe on admission, and the results for nonsevere cases on admission that became severe during hospitalization would continuously increase (i.e., blood leukocyte count, neutrophil count, C-reactive protein level, D-dimer level, alanine aminotransferase activity, aspartate aminotransferase activity, a-hydroxybutyrate dehydrogenase activity, lactate dehydrogenase activity and creatine kinase activity) or decrease (i.e., lymphocyte count) before reaching the peak or valley. This would cause a difference between the nonsevere and severe groups, enabling the identification of risk factors using statistical methods. Furthermore, the utility of a fixed value of an indicator on admission is only a component of that indicator at its highest or lowest level during hospitalization. In addition, under the same conditions, the risk factors in Table 3 might account for more severe cases than those in Table 4, as the difference between the nonsevere group and the severe group was larger for the indicators in Table 3. This view was demonstrated in our study. For example, in Table 3 , the $>10^{\star} 10^{\wedge} 9 / \mathrm{L}$ group accounted for $73.5 \%$ of all 34 severe cases; however, in Table 4 , the $>10^{*} 10^{\wedge} 9 / \mathrm{L}$ group only accounted for $14.7 \%$ of all 34 severe cases. Therefore, in theory, the conclusions would be more reliable when the highest or lowest level of the indicator during hospitalization was used compared with the fixed value of it on admission. Furthermore, it is easy to understand that more attention should be paid to patients whose indicators continue to increase (i.e., blood leukocyte count, neutrophil count, C-reactive protein level, D-dimer level, alanine aminotransferase activity, aspartate aminotransferase activity, a-hydroxybutyrate dehydrogenase activity, lactate dehydrogenase activity and creatine kinase activity) or decrease (i.e., lymphocyte count) during hospitalization.

Below, we discuss the clinical significance of these risk factors.

Leukocytes, such as neutrophils, lymphocytes and monocytes, are immune system components. When the human body is infected with viruses, the lymphocyte count usually remains normal or increases; in contrast, the neutrophil count decreases. However, in our study, lymphopenia and neutrocytosis were common in patients with severe COVID-19, reflecting dysregulation of the immune response.

Neutrocytosis is related to neutrophil extracellular traps (NETs), which originate from decondensed chromatin released to immobilize pathogens and can trigger immunothrombosis. ${ }^{6}$ In addition, postmortem biopsies from four COVID-19 patients revealed neutrophilic infiltration. ${ }^{7}$ As suggested by a previous study, neutrocytosis might be in part caused by a dysregulated myeloid cell compartment. Severe COVID-19 is marked by the occurrence of neutrophil precursors, as evidence of emergency myelopoiesis and dysfunctional mature neutrophils. ${ }^{8}$

Lymphopenia, a marker of impaired cellular immunity, is a cardinal laboratory finding reported in $67-90 \%$ of patients with COVID-19, with prognostic association in the vast majority of studies published to date. ${ }^{1-}$ 
${ }^{3}$ Several mechanisms likely contribute to the reduced number of T cells in the blood, including effects from the inflammatory cytokine milieu and T cell recruitment to sites of infection. ${ }^{9}$

C-reactive protein, a-hydroxybutyrate dehydrogenase and lactate dehydrogenase are serum inflammatory markers. Elevation in serum inflammatory markers reflects excessive inflammation and is predictive of subsequent critical illness and mortality in patients with COVID-19.2,3,10

D-dimer reflects coagulation function, whereby an increased D-dimer level indicates a hypercoagulable state, which might promote thrombus formation. Thrombotic complications were first reported in up to $30 \%$ of patients in intensive care units in China and the Netherlands. ${ }^{11,12}$ There is also emerging evidence of thrombosis in intravenous catheters and extracorporeal circuits and arterial vascular occlusive events, including acute myocardial infarction, acute limb ischemia, and stroke, in severe cases in the USA (United States of America), Italy and France. ${ }^{4}$

Alanine aminotransferase activity and aspartate aminotransferase activity are used to evaluate liver function. In patients critically ill with COVID-19, a hepatocellular injury pattern is detected in $14-53 \%$ of hospitalized patients. ${ }^{4}$ Aminotransferases are typically elevated but remain less than five times the upper limit of normal. Severe acute hepatitis has rarely been reported. ${ }^{4}$ In our study, we did not observe an obvious difference between the 40-80 U/L group and the $>80 \mathrm{U} / \mathrm{L}$ group, and most of the liver function alterations were temporary and reversible. Hence, liver function injury might be caused by multiple factors, including immunity, inflammation and drugs.

Renal function is often evaluated by creatinine levels. Acute kidney injury (AKI) is a frequent complication of COVID-19 and is associated with mortality. In China, the reported incidence of AKI in patients hospitalized with COVID-19 ranges from $0.5 \%$ to $29 \%$, occurring within a median of 7-14 days after admission. ${ }^{4}$ Studies from the USA have reported much higher rates of AKI. In fact, a study of nearly 5,500 patients with COVID-19 admitted to a New York City hospital system reported rates of AKI in $37 \%$, with $14 \%$ of these patients requiring dialysis. ${ }^{4}$ Nonetheless, SARS-CoV- 2 was not commonly found in urine, which means in most cases of kidney injury were probably not directly caused by SARS-CoV-2. ${ }^{13,14}$

Creatine kinase activity is used to assess cardiac function. SARS-CoV-2 can cause both direct and indirect cardiovascular sequelae, including myocardial injury, acute coronary syndromes, cardiomyopathy, acute cor pulmonale, arrhythmias, and cardiogenic shock, as well as the aforementioned thrombotic complications. ${ }^{15,16}$

\subsection{Administration of gamma globulin}

Administration of gamma globulin among our study cohort was not used as conventional therapy, and the wish of the patients and their relatives determined whether it was given.

As shown in Table 5, 34 severe cases were divided into two groups according to whether gamma globulin was used. Although gamma globulin administration appeared to be helpful for reducing mortality in 
severe cases, the $P$ value was greater than $0.05(P=0.180)$; thus, studies under the same conditions with larger samples are needed in the future. In fact, gamma globulin may be useful, as suggested by several studies. ${ }^{17,18}$

\section{Conclusion}

Compared with patients with only COVID-19, those with underlying diseases or coinfection other respiratory pathogens associated with community-acquired pneumonia showed a tendency to develop a severe case or to die.

In general, conclusions based on the highest or lowest level of an indicator during hospitalization are more reliable than those based on a fixed value on admission. It is easy to understand that more attention should be paid to patients whose blood markers continue to increase (i.e., blood leukocyte count, neutrophil count, C-reactive protein level, D-dimer level, alanine aminotransferase activity, aspartate aminotransferase activity, a-hydroxybutyrate dehydrogenase activity, lactate dehydrogenase activity and creatine kinase activity) or decrease (i.e., lymphocyte count) during hospitalization.

Multiple factors are related to severe COVID-19 and an outcome of death. Administration of gamma globulin may be helpful for reducing the mortality of severe COVID-19. More related studies are needed in the future.

\section{Declarations}

\section{Conflict of interest}

The Authors declare that there is no conflict of interest.

\section{Funding}

This research received no specific grant from any funding agency in the public, commercial, or not-forprofit sectors.

\section{Availability of data and materials}

All data generated or analyzed during this study are included in this published article [and its supplementary information files].

\section{References}

1. Guan WJ, Ni ZY, Hu Y, et al. Clinical characteristics of coronavirus disease 2019 in China. N Engl J Med 2020; 382: 1708-1720.

2. Zhou F, Yu T, Du R, et al. Clinical course and risk factors for mortality of adult inpatients with COVID19 in Wuhan, China: a retrospective cohort study. Lancet 2020; 395: 1054-1062. 
3. Zhang G, Zhang J, Wang B, et al. Analysis of clinical characteristics and laboratory findings of 95 cases of 2019 novel coronavirus pneumonia in Wuhan, China: a retrospective analysis. Respir Res 2020; $21: 74$.

4. Gupta A, Madhavan MV, Sehgal K, et al. Extrapulmonary manifestations of COVID-19. Nat Med 2020; 26: $1017-1032$.

5. Al-Lami RA, Urban RJ, Volpi E, et al. Sex hormones and novel corona virus infectious disease (COVID19). Mayo Clin Proc 2020; 95: 1710-1714.

6. Middleton EA, He XY, Denorme F, et al. Neutrophil extracellular traps contribute to immunothrombosis in COVID-19 acute respiratory distress syndrome. Blood 2020; 136: 1169-1179.

7. Tian S, Xiong Y, Liu H, et al. Pathological study of the 2019 novel coronavirus disease (COVID-19) through postmortem core biopsies. Mod Pathol 2020; 33: 1007-1014.

8. Schulte-Schrepping J, Reusch N, Paclik D, et al. Severe COVID-19 is marked by a dysregulated myeloid cell compartment. Cell 2020; 182: 1419-1440.e23.

9. Vabret N, Britton GJ, Gruber C, et al. Immunology of COVID-19: current state of the science. Immunity 2020; 52: 910-941.

10. Ruan Q, Yang K, Wang W, et al. Correction to: clinical predictors of mortality due to COVID-19 based on an analysis of data of 150 patients from Wuhan, China. Intensive Care Med 2020; 46: 12941297.

11. Cui S, Chen S, Li X, et al. Prevalence of venous thromboembolism in patients with severe novel coronavirus pneumonia. J Thromb Haemost 2020; 18: 1421-1424.

12. Klok FA, Kruip M, van der Meer NJM, et al. Incidence of thrombotic complications in critically ill ICU patients with COVID-19. Thromb Res 2020; 191: 145-147.

13. Puelles VG, Lütgehetmann M, Lindenmeyer MT, et al. Multiorgan and renal tropism of SARS-CoV-2. N Engl J Med 2020; 383: 590-592.

14. Ling $Y, X u S B$, Lin $Y X$, et al. Persistence and clearance of viral RNA in 2019 novel coronavirus disease rehabilitation patients. Chin Med J (Engl) 2020; 133: 1039-1043.

15. Driggin E, Madhavan MV, Bikdeli B, et al. Cardiovascular considerations for patients, health care workers, and health systems during the COVID-19 pandemic. J Am Coll Cardiol 2020; 75: 23522371.

16. Clerkin KJ, Fried JA, Raikhelkar J, et al. COVID-19 and cardiovascular disease. Circulation 2020; 141: 1648-1655.

17. Basic-Jukic N. Can hyperimmune anti-CMV globulin substitute for convalescent plasma for treatment of COVID-19? Med Hypotheses 2020; 144: 109903.

18. Sun L, Shen L, Fan J, et al. Clinical features of patients with coronavirus disease 2019 from a designated hospital in Beijing, China. J Med Virol 2020; DOI: 10.1002/jmv.25966.

\section{Figures}


186 confirmed cases with COVID-19

$\downarrow$

14 cases with severe underlying diseases were excluded

$\downarrow$

22 cases with common bacteria or viruses associated with community-acquired pneumonia were excluded

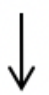

16 cases with both severe underlying diseases and other respiratory pathogens infection were excluded

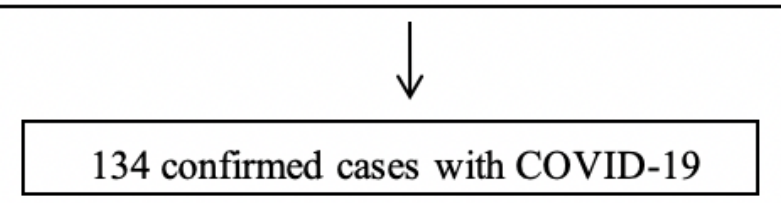

\section{Figure 1}

A flow chart, from the total number of patients to the 134 included in the study. Fourteen patients with severe underlying diseases (i.e., chronic lung disease, chronic heart disease, chronic liver disease, chronic kidney disease) were excluded. Twenty-two patients coinfected with 2019 novel coronavirus and other respiratory pathogens (i.e., bacteria, Chlamydia pneumoniae, Mycoplasma pneumoniae, adenovirus, or respiratory syncytial virus) associated with community-acquired pneumonia were excluded. Sixteen patients with both severe underlying diseases and other respiratory pathogen infections were excluded. Ultimately, 134 confirmed cases of COVID-19 were included in our study. 
186 confirmed cases with COVID-19

$\downarrow$

14 cases with severe underlying diseases were excluded

$\downarrow$

22 cases with common bacteria or viruses associated with community-acquired pneumonia were excluded

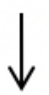

16 cases with both severe underlying diseases and other respiratory pathogens infection were excluded

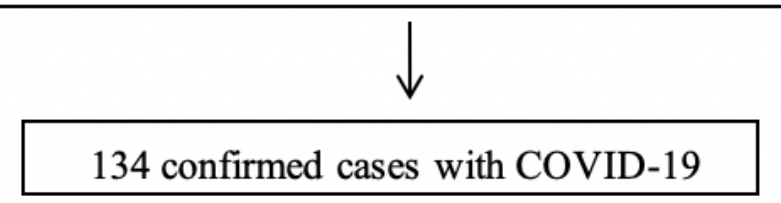

\section{Figure 1}

A flow chart, from the total number of patients to the 134 included in the study. Fourteen patients with severe underlying diseases (i.e., chronic lung disease, chronic heart disease, chronic liver disease, chronic kidney disease) were excluded. Twenty-two patients coinfected with 2019 novel coronavirus and other respiratory pathogens (i.e., bacteria, Chlamydia pneumoniae, Mycoplasma pneumoniae, adenovirus, or respiratory syncytial virus) associated with community-acquired pneumonia were excluded. Sixteen patients with both severe underlying diseases and other respiratory pathogen infections were excluded. Ultimately, 134 confirmed cases of COVID-19 were included in our study. 